\title{
ROOTS OF UNITY AS QUOTIENTS OF TWO ROOTS OF A POLYNOMIAL
}

\author{
ARTŪRAS DUBICKAS \\ (Received 1 March 2011; accepted 1 February 2012) \\ Communicated by I. E. Shparlinski \\ Dedicated to the memory of Alf van der Poorten
}

\begin{abstract}
Let $K$ be a number field. For $f \in K[x]$, we give an upper bound on the least positive integer $T=T(f)$ such that no quotient of two distinct $T$ th powers of roots of $f$ is a root of unity. For each $\varepsilon>0$ and each $f \in \mathbb{Q}[x]$ of degree $d \geq d(\varepsilon)$ we prove that $\log T(f)<(2+\varepsilon) \sqrt{d \log d}$. In the opposite direction, we show that the constant 2 cannot be replaced by a number smaller than 1 . These estimates are useful in the study of degenerate and nondegenerate linear recurrence sequences over a number field $K$.
\end{abstract}

2010 Mathematics subject classification: primary 11R04; secondary 11R18, $11 \mathrm{~B} 37$.

Keywords and phrases: root of unity, number field, linear recurrence sequence.

\section{Introduction}

Let $K$ be a number field, that is, a finite extension of the field of rational numbers $\mathbb{Q}$, and let

$$
f(x):=x^{d}+a_{d-1} x^{d-1}+\cdots+a_{0}=\prod_{i=1}^{d}\left(x-\alpha_{i}\right) \in K[x]
$$

be a monic polynomial of degree $d \geq 2$. It may happen that the quotient of two distinct roots of $f$, say, $\alpha_{i} / \alpha_{j}, 1 \leq i<j \leq d$, is a root of unity (for example, for $\left.f(x)=x^{d}-2 \in \mathbb{Q}[x]\right)$. We say that the polynomial $f$ is torsion-free if no quotient of two distinct roots of $f$ is a root of unity. In the case where $f(x)=\prod_{i=1}^{d}\left(x-\alpha_{i}\right)$ is not torsion-free, the polynomial $f_{T}(x):=\prod_{i=1}^{d}\left(x-\alpha_{i}^{T}\right)$ is torsion-free for some $T \in \mathbb{N}$. The smallest positive integer $T=T(f)$ with this property is called the torsion order of $f$, so that torsion-free polynomials have torsion order one. For example, for the polynomial $x^{d}-2$, we have $T\left(x^{d}-2\right)=d$. The torsion order of a polynomial can be greater than its degree, for example, $T\left(\Phi_{d}\right)=d$, where $\Phi_{d}$ is the $d$ th cyclotomic polynomial of degree $\varphi(d)$. Note that the torsion order $T(f)$ is independent of the field $K$. If $f \in K[x]$

(c) 2012 Australian Mathematical Publishing Association Inc. 1446-7887/2012 \$16.00 
is not monic and has a leading coefficient $a \in K \backslash\{0\}$ then the torsion order of $f$ is defined as $T(f / a)$.

Here is the main result of this paper.

Theorem 1. Let $K$ be a number field with $k:=[K: \mathbb{Q}]$. For every $f \in K[x]$ of degree $d \geq 2$,

$$
\log T(f)<(1.05314+\sqrt{6 k}) \sqrt{d \log (k d)} .
$$

Furthermore, if $\varepsilon>0$ then

$$
\log T(f)<(1+\varepsilon)(1+\sqrt{k}) \sqrt{d \log d}
$$

provided that $d$ is large enough. On the other hand, for all $\varepsilon>0$ there exists $d_{0}(\varepsilon)$ such that for each positive integer $d \geq d_{0}(\varepsilon)$ there is a monic polynomial $\Phi \in \mathbb{Z}[x] \subset K[x]$ of degree $d$ for which

$$
\log T(\Phi)>(1-\varepsilon) \sqrt{d \log d} .
$$

On replacing $\varepsilon$ by $\varepsilon / 2$ in (2) and selecting $K:=\mathbb{Q}$, we find that

$$
\exp ((1-\varepsilon) \sqrt{d \log d})<\max _{f \in \mathbb{Q}[x], \operatorname{deg} f=d} T(f)<\exp ((2+\varepsilon) \sqrt{d \log d})
$$

for each $\varepsilon>0$ and each sufficiently large $d$. Note that the difference between the upper and lower bounds in (4) is only in the constants 1 and 2.

The torsion order $T$ of the polynomial $f(x)=x^{d}+a_{d-1} x^{d-1}+\cdots+a_{0}$ is related to the properties of the linear recurrence sequence

$$
x_{n+d}=-a_{d-1} x_{n+d-1}-\cdots-a_{0} x_{n},
$$

where $n=1,2, \ldots$ Such a sequence is called degenerate if its characteristic polynomial $f$ has a pair of distinct roots whose ratio is a root of unity and nondegenerate otherwise. On replacing $f$ by $f_{T}$, where $T$ is the torsion order of $f$, one reduces the study of arbitrary linear recurrence sequences to the study of nondegenerate sequences. Theorem 1.2 in [4] asserts that, for some $t \leq T(f)$, each subsequence $x_{t n+s}$, where $n=1,2, \ldots$, is either identically zero or nondegenerate. Other applications of the torsion order to the Skolem-Mahler-Lech theorem were mentioned by Berstel and Mignotte [2] (see also [4, Ch. 2]). Schinzel [11] used $T(f)$ to treat an old problem of Pólya [9] on the description of rational functions $\sum_{n=0}^{\infty} u_{n} x^{n} \in \mathbb{Q}(x)$ whose numerators are divisible by only finitely many primes. In this sense the bound in (4) gives the best possible (up to a constant) estimate in [11, Theorem 2]. See also [14], where the case $K=\mathbb{Q}$ was considered. Robba [10] investigated the case of a number field, but his upper bound $T(f) \leq 2^{k d+1}$ is weaker than that given in (1).

It seems likely that if $f \in \mathbb{Q}[x]$ is irreducible over the field $\mathbb{Q}$ then the upper bound for $T(f)$ should be given by the inequality

$$
\varphi(T(f)) \leq d,
$$

where $\varphi$ is Euler's function. Note that equality holds in (5) for cyclotomic polynomials $f=\Phi_{d}$. Since $\lim \inf _{n \rightarrow \infty}(\varphi(n) \log \log n) / n=e^{-\gamma}$, where $\gamma=0.57721 \ldots$ is Euler's 
constant (see, for example, [5, Theorem 324]), the upper bound (5) via [11, Lemma 3] would imply the inequality

$$
T(f)<e^{\gamma} d(\log \log d+4)
$$

for every $d \geq 2$. Unfortunately, the proof of Corollary 2.1 (which is equivalent to (5)) in our paper [3] contains an error. The best known bound in this direction is due to Schinzel [11] who showed that

$$
T(f)<e^{3 \gamma / 2} d^{3 / 2}(\log \log d+4)^{3 / 2}
$$

for every irreducible polynomial $f \in \mathbb{Q}[x]$ of degree $d \geq 2$ (which is short of the conjectured bound (6)). In [1, 3, 6, 14], one can find different proofs of the inequality

$$
[K(\zeta): K] \leq d=[K(\alpha): K]
$$

when $\alpha, \alpha^{\prime}$ are two algebraic numbers conjugate over $K$ and $\zeta:=\alpha / \alpha^{\prime}$ is a root of unity. This shows that one can get rid of at least one root of unity among the ratios of roots of an irreducible polynomial $f \in K[x]$ of degree $d$ by taking some power $t$ for which $\varphi(t) \leq d$.

In the next section we shall prove (3) and give another example which shows that for some $f \in K[x]$ the torsion degree $T(f)$ may tend to infinity as $k \rightarrow \infty$ for cyclotomic extensions $K$ of $\mathbb{Q}$. In Section 3 we state two results on the least common multiple of positive integers $b_{1}, b_{2}, \ldots, b_{m}$, whose sum (or the sum of the values of Euler's function $\left.\varphi\left(b_{1}\right), \varphi\left(b_{2}\right), \ldots, \varphi\left(b_{m}\right)\right)$ does not exceed some fixed integer $n$. We give two more lemmas in Section 4, and then complete the proofs of (1) and (2) in Section 5.

\section{Examples}

Let $X(d)$ be the largest integer for which

$$
\sum_{p \leq X(d)}(p-1) \leq d,
$$

where the sum is taken over prime numbers $p$. Set

$$
\Phi(x):=g(x) \prod_{p \leq X(d)}\left(x^{p-1}+\cdots+x+1\right),
$$

where $g(x):=\prod_{i=1}^{e}(x-i) \in \mathbb{Z}[x]$ is a polynomial of degree

$$
e:=d-\sum_{p \leq X(d)}(p-1) .
$$

Then $\Phi(x) \in \mathbb{Z}[x]$ is a monic polynomial of degree $d$. (Note that $\Phi(x) \in \mathbb{Z}[x] \subset K[x]$ for every number field $K$.) Put

$$
Y(d):=\prod_{p \leq X(d)} p
$$


where the product is taken over primes $p$. Clearly, $\Phi_{T}$ is a torsion-free polynomial for $T=Y(d)$, since the $T$ th powers of the roots of $\Phi$ are $i^{T}$, where $i=1, \ldots, e$, and 1 , because $\zeta_{p}^{j Y(d)}=1$ whenever $p \leq X(d)$ and $j=1, \ldots, p-1$. (Throughout, $\zeta_{r}:=e^{2 \pi i / r}$ is the primitive $r$ th root of unity.) On the other hand, if some prime $p \leq X(d)$ does not divide $T$ then $\Phi_{T}$ is not torsion-free, because the quotient of two distinct roots of $\Phi_{T}$, say $\zeta_{p}^{2 T}$ and $\zeta_{p}^{T}$, is a root of unity. Consequently, for the polynomial defined in (8),

$$
T(\Phi)=Y(d) .
$$

Now, using the prime number theorem $p_{i} \sim i \log i$ as $i \rightarrow \infty$, from (7) and (9) one can easily derive that

$$
X(d) \sim \sqrt{d \log d} \text { and } \log Y(d) \sim X(d)
$$

as $d \rightarrow \infty$. Hence the inequality $\log T(\Phi)=\log Y(d)>(1-\varepsilon) \sqrt{d \log d}$ holds for every $d \geq d_{0}(\varepsilon)$ and every $\varepsilon>0$. This proves (3).

Suppose next that $K=\mathbb{Q}\left(\zeta_{m}\right)$, where $\varphi(m)=k$, so that $k=[K: \mathbb{Q}]$. Let us take

$$
G(x):=g_{1}(x) \prod_{m<p \leq X_{1}(d)}\left(x^{p}-\zeta_{m}\right),
$$

where the product is taken over primes $p$, the number $X_{1}(d)$ is the largest integer for which $\sum_{m<p \leq X_{1}(d)} p \leq d$, and $g_{1}(x):=\prod_{i=1}^{e_{1}}(x-i)$ with $e_{1}:=d-\sum_{m<p \leq X_{1}(d)} p$. By the same argument as above, we see that $G \in K[x]$ is a monic polynomial of degree $d$ whose torsion order is

$$
T(G)=m \prod_{m<p \leq X_{1}(d)} p>m \exp ((1-\varepsilon) \sqrt{d \log d})
$$

for each $\varepsilon>0$ and each sufficiently large $d$. Since $m>k$, it follows that

$$
\log T(G)>\log k+(1-\varepsilon) \sqrt{d \log d}
$$

for each $\varepsilon>0$ and each sufficiently large $d$.

\section{Two results for the least common multiple}

LeMma 2. Let $b_{1}, \ldots, b_{m}$ be some positive integers satisfying $b_{1}+\cdots+b_{m} \leq n$, where $n \geq 2$. Then

$$
\operatorname{lcm}\left(b_{1}, \ldots, b_{m}\right)<\exp (1.05314 \sqrt{n \log n}),
$$

where $1 \mathrm{~cm}$ stands for the least common multiple. Furthermore, for each $\varepsilon>0$ there is an integer $n_{0}(\varepsilon)$ such that, for every $n \geq n_{0}(\varepsilon)$,

$$
\operatorname{lcm}\left(b_{1}, \ldots, b_{m}\right)<\exp ((1+\varepsilon) \sqrt{n \log n}) .
$$

The first part of Lemma 2 was proved by Massias [7]. In fact, $\operatorname{lcm}\left(b_{1}, \ldots, b_{m}\right)$ is the order of an element consisting of $m$ disjoint cycles of lengths $b_{1}, \ldots, b_{m}$ in the 
full symmetric group on $n$ elements $S_{n}$. Let $M(n)$ be the maximal order of an element in $S_{n}$. Landau proved that $\log M(n) \sim \sqrt{n \log n}$ as $n \rightarrow \infty$ and Shah [12] gave a more precise asymptotic formula

$$
\log M(n)=\sqrt{n \log n}(1+\log \log n /(2 \log n)+O(1 / \log n)) .
$$

This implies the second part of Lemma 2. See also [8, 13] for further work on this problem. It is interesting to note that the upper bound $1.05313 \ldots$ is attained for the symmetric group $S_{1319766 \text {. }}$

The following lemma was proved by Berstel and Mignotte [2].

Lemma 3. Let $b_{1}, \ldots, b_{m}$ be positive integers satisfying $\varphi\left(b_{1}\right)+\cdots+\varphi\left(b_{m}\right) \leq n$, where $n \geq 2$. Then

$$
\operatorname{lcm}\left(b_{1}, \ldots, b_{m}\right)<\exp (\sqrt{6 n \log n}) .
$$

Furthermore, the constant $\sqrt{6}$ can be replaced by any constant strictly greater than 1 if $n$ is sufficiently large.

\section{Some divisibility results for the torsion order of a polynomial}

Let $f \in K[x]$ be a separable polynomial of degree $d \geq 2$. We say that two of its roots $\alpha$ and $\alpha^{\prime}$ belong to the same equivalence class if their quotient is a root of unity. Suppose that there are $s:=s(f)$ distinct equivalence classes. It is easy to see that each equivalence class contains the same number of elements, say, $\ell:=\ell(f)$ roots of $f$, so

$$
d=s \ell=s(f) \ell(f) .
$$

Now, let $f \in K[x]$ be an irreducible polynomial of degree $d \geq 2$, and let $\alpha$ be one of its roots. Suppose that $r$ is the largest positive integer for which

$$
\mathbb{Q}\left(\zeta_{r}\right) \subseteq K(\alpha) .
$$

Then $K\left(\zeta_{r}\right) \subseteq K(\alpha)$. Since $K\left(\zeta_{r}\right)$ is a normal extension of $K$, the field $K\left(\zeta_{r}\right)$ is contained in the field $K\left(\alpha^{\prime}\right)$ for any conjugate $\alpha^{\prime}$ of $\alpha$ over $K$. Hence $\mathbb{Q}\left(\zeta_{r}\right)$ is contained in every field $K\left(\alpha^{\prime}\right)$ too. Thus $r:=r(f, K)$ is independent of the choice of $\alpha$ and depends only on the polynomial $f$ and the field $K$. Of course,

$$
r(f, K)=w(K(\alpha)),
$$

where $w(F)$ stands for the number of distinct roots of unity lying in the field $F$.

We claim that if $\alpha \neq \alpha^{\prime}$ and $\alpha / \alpha^{\prime}$ is a root of unity (so that $\ell \geq 2$ ) then

$$
\alpha^{\ell r}=\left(\alpha^{\prime}\right)^{\ell r} \text {. }
$$

Indeed, write $\gamma(C)$ for the product of conjugates of $\alpha$ belonging to the equivalence class $C$. From $\alpha, \alpha^{\prime} \in C$ we obtain $\alpha^{\ell}=\zeta \gamma(C)$ and $\left(\alpha^{\prime}\right)^{\ell}=\zeta^{\prime} \gamma(C)$ for some roots of unity $\zeta, \zeta^{\prime}$. It is clear that $s=s(f)$ equivalence classes are blocks of imprimitivity of the Galois group $\operatorname{Gal}(K(\alpha) / K)$. Hence every automorphism $\sigma \in \operatorname{Gal}(K(\alpha) / K)$ satisfying $\sigma(\alpha)=\alpha$ is a permutation of the set $C$, so it maps $\gamma(C)$ to $\gamma(C)$. Thus $\gamma(C) \in K(\alpha)$. 
This yields $\zeta=\alpha^{\ell} / \gamma(C) \in K(\alpha)$, so that $\zeta \in \mathbb{Q}\left(\zeta_{r}\right)$, by (11). Hence $\zeta^{r}=1$. By the same argument, $\left(\zeta^{\prime}\right)^{r}=1$. Therefore,

$$
\left(\frac{\alpha}{\alpha^{\prime}}\right)^{\ell r}=\left(\frac{\zeta \gamma(C)}{\zeta^{\prime} \gamma(C)}\right)^{r}=\frac{\zeta^{r}}{\left(\zeta^{\prime}\right)^{r}}=1
$$

This proves (13) and so implies the following result.

Lemma 4. The torsion order of an irreducible polynomial $f \in K[x]$ satisfies

$$
T(f) \mid \ell(f) r,
$$

where $r:=r(f, K)$ is defined in (11) and (12).

Next we extend this result to the product of two irreducible polynomials.

Lemma 5. Let $f_{1}, f_{2} \in K[x]$ be two distinct irreducible polynomials having $\ell_{1}:=\ell\left(f_{1}\right)$ and $\ell_{2}:=\ell\left(f_{2}\right)$ elements in their equivalence classes and with $r_{1}:=r\left(f_{1}, K\right)$ and $r_{2}:=r\left(f_{2}, K\right)$ defined as in (11) and (12). Then

$$
T\left(f_{1} f_{2}\right) \mid \operatorname{lcm}\left(\ell_{1} \operatorname{lcm}\left(r_{1}, r_{2}\right), \ell_{2} \operatorname{lcm}\left(r_{1}, r_{2}\right)\right) \text {. }
$$

Proof. In view of Lemma 4 it suffices to prove that if $\alpha$ is a root of $f_{1}$ and $\beta$ is a root of $f_{2}$ such that $\alpha / \beta$ is a root of unity then

$$
\alpha^{L}=\beta^{L},
$$

where $L:=\operatorname{lcm}\left(\ell_{1} r, \ell_{2} r\right)$ and $r:=\operatorname{lcm}\left(r_{1}, r_{2}\right)$. Suppose that $\alpha$ and $\beta$ belong to the equivalence classes $C_{1}$ and $C_{2}$. As above, let $\gamma\left(C_{1}\right)$ and $\gamma\left(C_{2}\right)$ be the product of conjugates of $\alpha$ from $C_{1}$ and the product of conjugates of $\beta$ from $C_{2}$. Then

$$
\alpha^{\ell_{1}}=\xi_{1} \gamma\left(C_{1}\right) \text { and } \beta^{\ell_{2}}=\xi_{2} \gamma\left(C_{2}\right)
$$

with some roots of unity $\xi_{1}, \xi_{2}$.

Let $S$ be the set of $\operatorname{deg} f_{1}+\operatorname{deg} f_{2}$ roots of the polynomial $f_{1} f_{2}$. The equivalence class of $S$ containing $\alpha$ consists of $C_{1} \cup C_{2}$. Consider the Galois group $\operatorname{Gal}(K(\alpha, \beta) / K)$ as permutations of the set $S$. Each element $\sigma \in \operatorname{Gal}(K(\alpha, \beta) / K)$ that fixes $\alpha$ (or $\beta$ ) permutes the class $C_{1}$ and the class $C_{2}$. Hence the products $\gamma\left(C_{1}\right)$ and $\gamma\left(C_{2}\right)$ both lie in the intersection $K(\alpha) \cap K(\beta)$. Thus $\xi_{1} \in K(\alpha)$ and $\xi_{2} \in K(\beta)$, by (14). From (14), the quotient $\zeta:=\gamma\left(C_{1}\right)^{L / \ell_{1} r} / \gamma\left(C_{2}\right)^{L / \ell_{2} r}$ is a root of unity, because $\alpha / \beta$ is a root of unity. Note that $\zeta, \xi_{1} \in K(\alpha)$ yields $\zeta, \xi_{1} \in \mathbb{Q}\left(\zeta_{r_{1}}\right)$, by the definition of $r_{1}$. Similarly, $\zeta, \xi_{2} \in K(\beta)$ yields $\zeta, \xi_{2} \in \mathbb{Q}\left(\zeta_{r_{2}}\right)$. It follows that $\zeta, \xi_{1}, \xi_{2}$ lie in the compositum

$$
\mathbb{Q}\left(\zeta_{r_{1}}\right) \mathbb{Q}\left(\zeta_{r_{2}}\right)=\mathbb{Q}\left(\zeta_{r_{1}}, \zeta_{r_{2}}\right)=\mathbb{Q}\left(\zeta_{\mathrm{lcm}\left(r_{1}, r_{2}\right)}\right)=\mathbb{Q}\left(\zeta_{r}\right) .
$$

Now, using the fact that the root of unity $\zeta \xi_{1}^{L / \ell_{1} r} \xi_{2}^{-L / \ell_{2} r}$ belongs to the field $\mathbb{Q}\left(\zeta_{r}\right)$ (so that its $r$ th power is 1 ), we obtain

$$
\frac{\alpha^{L}}{\beta^{L}}=\frac{\xi_{1}^{L / \ell_{1}} \gamma\left(C_{1}\right)^{L / \ell_{1}}}{\xi_{2}^{L / \ell_{2}} \gamma\left(C_{2}\right)^{L / \ell_{2}}}=\left(\frac{\xi_{1}^{L / \ell_{1} r} \zeta}{\xi_{2}^{L / \ell_{2} r}}\right)^{r}=1,
$$

which completes the proof of the lemma. 


\section{Proof of the upper bound for torsion order}

Without loss of generality, we may assume that $f \in K[x]$ is monic. Let us write $f$ in the form

$$
f(x)=f_{1}(x)^{k_{1}} f_{2}(x)^{k_{2}} \cdots f_{m}(x)^{k_{m}}
$$

for distinct irreducible monic polynomials $f_{1}, \ldots, f_{m} \in K[x]$ and $k_{1}, \ldots, k_{m} \in \mathbb{N}$. Obviously, $T(f)=T\left(f_{1} \cdots f_{m}\right)$ and the degree of the polynomial $f_{1} \cdots f_{m}$ is smaller than $\operatorname{deg} f$ provided that at least one $k_{i}$ is greater than 1 . So we may assume that $k_{1}=\cdots=k_{m}=1$. Then, by Lemmas 4 and 5 , for $f=f_{1} \cdots f_{m}$,

$$
T(f) \mid \operatorname{lcm}\left(\ell_{1}, \ldots, \ell_{m}\right) \operatorname{lcm}\left(r_{1}, \ldots, r_{m}\right)
$$

with $r_{i}:=r\left(f_{i}, K\right)$ defined in (11) and (12), because $\operatorname{lcm}\left(\ell_{i} \operatorname{lcm}\left(r_{i}, r_{j}\right), \ell_{j} \operatorname{lcm}\left(r_{i}, r_{j}\right)\right)$ divides $\operatorname{lcm}\left(\ell_{i}, \ell_{j}\right) \operatorname{lcm}\left(r_{i}, r_{j}\right)$ for all indices $i, j$ in the range $1 \leq i, j \leq m$. From (10) we see that $\ell_{i} \mid d_{i}$, where $d_{i}=\operatorname{deg} f_{i}$. Thus $\operatorname{lcm}\left(\ell_{1}, \ldots, \ell_{m}\right)$ divides $\operatorname{lcm}\left(d_{1}, \ldots, d_{m}\right)$. Using the equality $\sum_{i=1}^{m} d_{i}=d=\operatorname{deg} f$ from Lemma 2 we deduce that

$$
\operatorname{lcm}\left(\ell_{1}, \ldots, \ell_{m}\right) \leq \operatorname{lcm}\left(d_{1}, \ldots, d_{m}\right)<\exp (1.05314 \sqrt{d \log d}) .
$$

Suppose that $\alpha_{i}$ is a root of $f_{i}$ for $i=1, \ldots, m$. Then we see that $\mathbb{Q}\left(\zeta_{r_{i}}\right) \subseteq K\left(\alpha_{i}\right)$ from $(11)$. Hence $\left[\mathbb{Q}\left(\zeta_{r_{i}}\right): \mathbb{Q}\right]=\varphi\left(r_{i}\right)$ divides

$$
\left[K\left(\alpha_{i}\right): \mathbb{Q}\right]=[K: \mathbb{Q}] \cdot\left[K\left(\alpha_{i}\right): K\right]=k d_{i} .
$$

In particular, $\varphi\left(r_{i}\right) \leq k d_{i}$ for each $i=1, \ldots, m$ and thus

$$
\sum_{i=1}^{m} \varphi\left(r_{i}\right) \leq k \sum_{i=1}^{m} d_{i}=k d .
$$

Therefore, Lemma 3 implies that

$$
\operatorname{lcm}\left(r_{1}, \ldots, r_{m}\right)<\exp (\sqrt{6 k d \log (k d)})
$$

Now, from (15), multiplying (16) and (17) we derive (1).

Next, suppose that $\varepsilon>0$ and that $d$ is large enough. Then, by Lemmas 2 and 3 , inequality (16) becomes

$$
\operatorname{lcm}\left(\ell_{1}, \ldots, \ell_{m}\right) \leq \operatorname{lcm}\left(d_{1}, \ldots, d_{m}\right)<\exp ((1+\varepsilon) \sqrt{d \log d}),
$$

whereas (17) is replaced by

$$
\operatorname{lcm}\left(r_{1}, \ldots, r_{m}\right)<\exp ((1+\varepsilon / 2) \sqrt{k d \log (k d)})<\exp ((1+\varepsilon) \sqrt{k d \log d}) .
$$

Multiplying these two inequalities, we derive (2) in view of (15). 


\section{References}

[1] M. G. Aschbacher and R. M. Guralnick, 'On Abelian quotients of primitive groups', Proc. Amer. Math. Soc. 107 (1989), 89-95.

[2] J. Berstel and M. Mignotte, 'Deux propriétés décidables des suites recurrentes linéaires', Bull. Soc. Math. France 104 (1976), 175-194.

[3] P. Drungilas and A. Dubickas, 'On subfields of a field generated by two conjugate algebraic numbers', Proc. Edinb. Math. Soc. 47 (2004), 119-123.

[4] G. Everest, A. van der Poorten, I. Shparlinski and T. Ward, Recurrence Sequences, Mathematical Surveys and Monographs, 104 (American Mathematical Society, Providence, RI, 2003).

[5] G. H. Hardy and E. M. Wright, An Introduction to the Theory of Numbers (Oxford University Press, Oxford, 1979).

[6] I. M. Isaacs, 'Quotients which are roots of unity (solution of problem 6523)', Amer. Math. Monthly 95 (1988), 561-562.

[7] J.-P. Massias, 'Majoration explicite de l'ordre maximum d'un élément du groupe symétrique', Ann. Fac. Sci. Toulouse Math. 6 (1984), 269-281.

[8] J.-P. Massias, J.-L. Nicolas and G. Robin, 'Evaluation asymptotique de l'ordre maximum d'un élément du groupe symétrique', Acta Arith. 104 (1988), 221-242.

[9] G. Pólya, 'Arithmetische Eigenschaften der Reihenentwicklungen rationaler Funktionen', J. reine angew. Math. 151 (1921), 1-31.

[10] Ph. Robba, 'Zéros de suites récurrentes linéaires', Groupe Étude Anal. Ultramétrique, 5 (1977/78), Exposé No. 13, 1978, 5 pp.

[11] A. Schinzel, 'Around Pólya's theorem on the set of prime divisors of a linear recurrence', in: Diophantine Equations. Papers from the international conference held in honor of T. N. Shorey's 60th birthday, Mumbai, 16-20 December 2005, (ed. N. Saradha) (Narosa Publishing House, New Delhi, 2008), pp. 225-233.

[12] S. Shah, 'An inequality for the arithmetical function $g(x)^{\prime}$, J. Indian Math. Soc. 3 (1939), 316-318.

[13] M. Szalay, 'On the maximal order in $S_{n}$ and $S_{n}^{*}$, Acta Arith. 37 (1980), 321-331.

[14] K. Yokoyama, Z. Li and I. Nemes, 'Finding roots of unity among quotients of the roots of an integral polynomial', in: Proceedings of the 1995 International Symposium on Symbolic and Algebraic Computation, ISSAC'95, Montreal, 10-12 July 1995, (ed. A. H. M. Levelt) (ACM Press, New York, 1995), pp. 85-89.

\section{ARTŪRAS DUBICKAS, Department of Mathematics and Informatics, Vilnius University, Naugarduko 24, Vilnius LT-03225, Lithuania e-mail: arturas.dubickas@mif.vu.lt}

